\title{
RESEARCH
}

Open Access

\section{Prescribers' satisfaction with delivering medications for opioid use disorder}

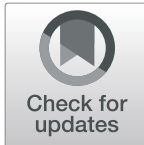

Hannah K. Knudsen ${ }^{1 *}$, Randy Brown², Nora Jacobson ${ }^{3}$, Julie Horst ${ }^{4}$, Jee-Seon Kim5 ${ }^{5}$ Hanna Kim5 ${ }^{5}$ Lynn M. Madden ${ }^{6}$, Eric Haram ${ }^{7}$ and Todd Molfenter ${ }^{4}$

\begin{abstract}
Background: Expanding access to medications for opioid use disorder (MOUD), such as buprenorphine and extended release (XR) naltrexone, is critical to addressing the US opioid epidemic, but little is known about prescriber satisfaction with delivering these two types of MOUD. The current study describes the satisfaction of prescribers delivering buprenorphine and XR-naltrexone while examining whether satisfaction is associated with current patient census and organizational environment.
\end{abstract}

Methods: As part of a cluster randomized clinical trial (RCT) focused on expanding access to medication for opioid use disorder, 41 MOUD prescribers in Florida, Ohio, and Wisconsin completed a web-based survey. The survey included measures of prescriber satisfaction with delivering buprenorphine treatment and XR-naltrexone. In addition, the survey measured several prescriber characteristics and their perceptions of the organizational environment.

Results: Prescribers were generally satisfied with their work in delivering these two types of MOUD. Prescribers reporting a greater number of patients $(r=.46, p=.006)$, those who would recommend the center to others $(r=$ $.56, p<.001)$, and those reporting positive relationships with staff $(r=.56, \mathrm{p}<.001)$ reported significantly greater overall satisfaction with delivering buprenorphine treatment. Prescribers who more strongly endorsed feeling overburdened reported lower overall buprenorphine satisfaction $(r=-.37, p=.02)$. None of the prescriber characteristics or perceptions of the organizational environment were significantly associated with overall satisfaction with delivering XR-naltrexone treatment.

Conclusions: The generally high levels of satisfaction with both types of MOUD is notable given that prescriber dissatisfaction can lead to turnover and impact intentions to leave the profession. Future research should continue to explore the prescriber characteristics and organizational factors associated with satisfaction in providing different types of MOUD.

Registration: ClinicalTrials.gov. NCT02926482. Date of registration: September 9, 2016. https://clinicaltrials.gov/ct2/ show/NCT02926482.

Keywords: prescriber satisfaction, buprenorphine, extended-release naltrexone, opioid use disorder

\footnotetext{
*Correspondence: hannah.knudsen@uky.edu

'Department of Behavioral Science and Center on Drug and Alcohol

Research, University of Kentucky, 845 Angliana Ave., Room 204, KY 40508 Lexington, USA

Full list of author information is available at the end of the article
}

(c) The Author(s). 2021 Open Access This article is licensed under a Creative Commons Attribution 4.0 International License, which permits use, sharing, adaptation, distribution and reproduction in any medium or format, as long as you give appropriate credit to the original author(s) and the source, provide a link to the Creative Commons licence, and indicate if changes were made. The images or other third party material in this article are included in the article's Creative Commons licence, unless indicated otherwise in a credit line to the material. If material is not included in the article's Creative Commons licence and your intended use is not permitted by statutory regulation or exceeds the permitted use, you will need to obtain permission directly from the copyright holder. To view a copy of this licence, visit http://creativecommons.org/licenses/by/4.0/ The Creative Commons Public Domain Dedication waiver (http://creativecommons.org/publicdomain/zero/1.0/) applies to the data made available in this article, unless otherwise stated in a credit line to the data. 


\section{Background}

The US opioid epidemic continues to be a major public health problem, resulting in nearly 450,000 deaths from 1999 to 2018 [1], with concerns growing amid early evidence that the COVID-19 pandemic is worsening this crisis [2-4]. Limited access to evidence-based treatment, particularly medications for opioid use disorder (MOUD) $[5,6]$, continues to be a significant challenge despite efforts to expand treatment access [7-9]. If access to MOUD is to expand in the US, it is critically important to continue to expand the number of medical professionals who are willing to prescribe buprenorphine and extended-release naltrexone (XR-naltrexone). Although the number of medical professionals who have the capacity to prescribe buprenorphine continues to grow [10-13], recent data indicate that only half of waivered prescribers have any patients receiving buprenorphine and that median monthly census is considerably lower than the cap on patients, even among those holding the 275-patient waiver [14]. Utilization of XRnaltrexone is even lower than buprenorphine [15], particularly in primary care [16].

An understudied issue is prescriber satisfaction with delivering these two types of MOUD. A systematic review published by Becker and Fiellin in 2005 [17] noted a gap in the literature regarding prescriber satisfaction with delivering buprenorphine treatment. Since that review, there have been two publications regarding prescriber satisfaction, one a qualitative study of rural prescribers [18] and the other a survey of a small sample of physicians in three states [19]. Andrilla and colleagues found that rural prescribers described their buprenorphine practice as rewarding and meaningful. However, a survey of waivered physicians indicated that prescribers rated their buprenorphine work as significantly less personally rewarding when compared to their general medical practice [19]. To date, there have been no studies of prescriber satisfaction with delivering XR-naltrexone as a treatment for OUD. These gaps in the literature are notable because there is a substantial literature regarding global physician satisfaction, which is associated with burnout, turnover intention, and intentions to leave the practice of medicine [20-22]. In addition, research has shown that physician satisfaction is associated with patients' satisfaction [23, 24] as well as patients' compliance with medical recommendations [25].

The lack of comparisons in delivering of buprenorphine versus extended-release naltrexone is notable given that implementation of the two medications may have some differences. For example, research has demonstrated major differences in the percentage of individuals who are successfully inducted onto the medication, with lower rates of successful induction for extendedrelease naltrexone than buprenorphine [26]. There is also some evidence of lower retention for extendedrelease naltrexone $[15,27]$, and that buprenorphine, but not extended-release naltrexone, being associated with lower odds of opioid-related overdose [28]. It is unknown if these challenges for initiation and retention translate into lower prescriber satisfaction with delivering extended-release naltrexone relative to buprenorphine satisfaction.

The aim of the current study was to expand on our prior work regarding buprenorphine satisfaction [19] by exploring the satisfaction of prescribers delivering buprenorphine as well as XR-naltrexone. In addition to considering satisfaction with delivering both types of MOUD, we sought to examine a new set of variables as potential correlates of MOUD satisfaction, including current MOUD patient census and prescribers' perceptions of the larger organization. Delivery of MOUD requires physicians to develop additional skills and competence, as OUD can be a challenging condition to treat. Patient census was conceived as a proxy measure for competence and perceived effectiveness, as presumably physicians would only be willing to take on more patients if they felt they had the capacity and confidence to effectively treat them. Similarly, tenure in the addiction field and addiction-specific board certifications may serve as indicators of competence, as greater years of experience and gaining specialized training in addiction should be positively correlated with more developed clinical skills. Previous research has shown that clinicians' perceived effectiveness is positively associated with job satisfaction [29]. Regarding the organizational environment, prior research on physician satisfaction has shown the importance of positive relationships among clinicians within organizations $[30,31]$ as well as the negative impacts of high job demands and burdens [29].

\section{Methods \\ Sample and data collection}

Data were drawn from a cluster randomized clinical trial (RCT) that sought to increase access to MOUD (NCT02926482) in 37 organizations in Florida, Ohio, and Wisconsin through a comparison of two sets of implementation strategies [32]. Within these 37 organizations, the study sample consisted of 61 treatment units within 31 specialty addiction treatment organizations and 14 treatment units within 6 health systems. Using procedures similar to a survey fielded in the first year of the study [19], during the third year of this project, the contact person for each organization participating in the RCT forwarded invitations to MOUD prescribers within these organizations to participate in a web-based survey. Multiple reminders were sent to potential participants about completing the survey, but no financial incentive for survey completion was provided. Forty-one 
respondents completed the survey from June 2019 to February 2020, representing 40 physicians and one nurse practitioner. The University of Wisconsin's institutional review board approved all study procedures.

\section{Measures}

Items measuring prescriber satisfaction with providing buprenorphine and XR-naltrexone treatment were adapted from the Physician Worklife Survey [33]. Among those providing each MOUD, five items asked about global satisfaction with their current buprenorphine work, and five items inquired about their current XR-naltrexone work. For each item, response options ranged from 1 ("strongly disagree") to 5 ("strongly agree"). In addition to considering each item separately, mean scales of buprenorphine job satisfaction $(\alpha=0.87)$ and XR-naltrexone job satisfaction $(\alpha=0.89)$ were calculated, after reverse-coding the negative valence items.

The survey measured several prescriber characteristics as well as their perceptions of the organizational environment. Prescribers indicated whether they were waivered to prescribe buprenorphine $(1=$ yes, $0=$ no $)$ and whether their site provided XR-naltrexone $(1=$ yes, $0=$ no). Waivered prescribers were asked about their current buprenorphine census (i.e., number of patients currently being treated with buprenorphine) and current waiver limit (i.e., 30 patients, 100 patients, or 275 patients). Using these two pieces of information, an additional variable was constructed regarding the percentage of the waiver currently used. For prescribers of XR-naltrexone, current census of patients being treated with XRnaltrexone was also reported. Prescribers were asked about the following addiction-focused board certifications: Addiction Medicine by the American Board of Addiction Medicine, Addiction Medicine by the American Board of Preventive Medicine, and Addiction Psychiatry by the American Board of Psychiatry and Neurology. Responses were coded into those with addiction board certification $(=1)$ and those without certification $(=0)$. Four items measured perceptions of the organizational environment, with responses ranging from 1 ("strongly disagree") to 5 ("strongly agree"). These items focused on organizational commitment, positive relationships with clinical staff, work overload, and perceived respect by the organization. Demographic characteristics included age, gender, and race $(0=$ white, $1=$ all others).

\section{Statistical analysis}

First, descriptive statistics were calculated for all measures. Due to the small sample, analyses relied upon Pearson's product-moment correlations between the measures of prescriber satisfaction and patient census, physician experience, and perceptions of the organizational environment. For the sub-sample who reported being waivered and prescribing XR-naltrexone, paired $t$-tests were used to test whether prescriber satisfaction differed between the two medications. Given the exploratory nature of the study, we did not correct for multiple comparisons. Data were collected during an organizational RCT in three states, so study condition of the prescribers' organization was examined using independent samples $t$-tests and the three states were compared by one-way analysis of variance. There were no significant differences in buprenorphine satisfaction or XR-naltrexone satisfaction by study condition or by state (results not shown).

\section{Results}

Among the 41 respondents, the average age was 51.0 $(\mathrm{SD}=11.0), 37.5 \%(\mathrm{n}=15)$ were female, and the majority identified as white $(70.7 \%, \mathrm{n}=29)$. About $37.5 \%(\mathrm{n}=$ 15) were board certified in an addiction specialty. Nearly all of the respondents held the waiver to prescribe buprenorphine $(92.7 \%, \mathrm{n}=38)$. Of the waivered prescribers, the most common waiver type was the 275patient waiver $(44.7 \%, \mathrm{n}=17) ; 31.6 \%$ held the 100 patient waiver $(\mathrm{n}=12)$, and $23.7 \%$ held the 30 -patient waiver $(n=9)$. The average census of current buprenorphine patients among waivered prescribers was 56.4 (SD $=57.0$ ), but the average proportion of their waiver being used was just $37.4 \%(\mathrm{SD}=24.8)$. About $78.1 \%$ of the sample $(\mathrm{n}=32)$ indicated that they provide XRnaltrexone for OUD, and for these prescribers, the average census of patients currently receiving XR-naltrexone for OUD was $20.7(\mathrm{SD}=26.3)$.

Table 1 presents satisfaction with buprenorphine work among waivered prescribers and satisfaction with XRnaltrexone work among those offering this medication to treat OUD. As seen in Table 1, physicians were generally satisfied with their work in delivering these two types of MOUD. Satisfaction measures with positive valences (e.g., personally rewarding) had means near 4.0, indicating agreement, while items with negative valences (e.g., frustration) had means near 2.0, indicating disagreement. For the sub-sample of prescribers offering both medications $(\mathrm{n}=31)$, paired $\mathrm{t}$-tests indicated there was only one significant difference in the five items, such that prescribers more strongly endorsed buprenorphine work being a source of frustration (mean $=2.3, \mathrm{SD}=$ 0.9 ) than XR-naltrexone work (mean $=2.0, \mathrm{SD}=0.8, t=$ $-2.52, p=.02$ ) (other results not shown).

Bivariate correlations between satisfaction with buprenorphine work, prescriber characteristics, and perceptions of the organizational environment are presented in Table 2. Current number of buprenorphine patients was associated with all five satisfaction measures, such that prescribers with greater numbers of patients more 
Table 1 Descriptive statistics of physician satisfaction with buprenorphine $(n=38)$ and XR-naltrexone work $(n=32)$

\begin{tabular}{lllll}
\hline & \multicolumn{2}{l}{ Buprenorphine Work } & \multicolumn{2}{l}{ XR-Naltrexone Work } \\
\hline & Mean (SD) & \% Agree or Strongly Agree (n) & Mean SD & \% Agree or Strongly Agree (n) \\
I find my present work personally rewarding. & $4.2(0.7)$ & $84.2 \%(32)$ & $4.1(0.9)$ & $81.3 \%(26)$ \\
Overall, I am pleased with my work. & $4.2(0.6)$ & $89.5 \%(34)$ & $4.1(0.8)$ & $87.5 \%(28)$ \\
Overall, I am satisfied with my current practice. & $4.1(0.7)$ & $83.8 \%(31)$ & $3.9(0.8)$ & $84.4 \%(27)$ \\
My current work is a major source of frustration. & $2.2(0.9)$ & $13.2 \%(5)$ & $2.0(0.8)$ & $3.1 \%(1)$ \\
My work in this practice has not met my expectations. & $2.1(0.8)$ & $7.9 \%(3)$ & $2.1(1.0)$ & $12.5 \%(4)$ \\
Mean satisfaction scale & $4.0(0.6)$ & & $4.0(0.7)$ & \\
\hline
\end{tabular}

Notes. Items were adapted from the Physician Worklife Survey (Williams et al., 1999), with response options ranging from $1=$ strongly disagree to $5=$ strongly agree. Negative valence items were reverse-coded for the mean scales

strongly endorsed the positive valence questions and reported lower frustration and unmet expectations. However, the measure of the proportion of waiver used was not associated with any of the buprenorphine satisfaction measures. Regarding perceptions of the organizational environment, prescribers who would recommend the treatment center to colleagues more strongly endorsed the positive valence items while reporting lower frustration and unmet expectations. Good relationships with clinical staff were also associated with the satisfaction items, with the exception of the item regarding unmet expectations. Feeling overburdened by clinical responsibilities was only associated with one of the five satisfaction items (i.e., satisfied with current practice), while feeling more valued by the organization was correlated with greater endorsement of buprenorphine work being rewarding and lower endorsement of frustration. There were no differences in satisfaction with buprenorphine work by tenure in the addiction field or by addiction-specific board certification.

Next, the buprenorphine work satisfaction items were combined into a mean scale of overall satisfaction, and the prescriber characteristics and perceptions of the

Table 2 Pearson's product-moment correlations between patient volume, prescriber characteristics, organizational environment, and buprenorphine satisfaction

\begin{tabular}{|c|c|c|c|c|c|}
\hline & $\begin{array}{l}\text { I find my present } \\
\text { buprenorphine } \\
\text { clinical work } \\
\text { personally rewarding. }\end{array}$ & $\begin{array}{l}\text { Overall, I am } \\
\text { pleased with my } \\
\text { buprenorphine } \\
\text { work. }\end{array}$ & $\begin{array}{l}\text { Overall I am } \\
\text { satisfied with my } \\
\text { current } \\
\text { buprenorphine } \\
\text { practice. }\end{array}$ & $\begin{array}{l}\text { My current } \\
\text { buprenorphine work } \\
\text { situation is a major } \\
\text { source of frustration. }\end{array}$ & $\begin{array}{l}\text { My work in this } \\
\text { buprenorphine } \\
\text { practice has not met } \\
\text { my expectations. }\end{array}$ \\
\hline $\begin{array}{l}\text { Current number of } \\
\text { buprenorphine patients }\end{array}$ & $0.36^{*}$ & $0.39^{*}$ & $0.35^{*}$ & $-0.41^{*}$ & $-0.34^{*}$ \\
\hline $\begin{array}{l}\text { Proportion of waiver used } \\
\text { (i.e., current number } \\
\text { divided by waiver limit) }\end{array}$ & 0.03 & 0.12 & 0.30 & -0.21 & -0.28 \\
\hline $\begin{array}{l}\text { Tenure in addiction } \\
\text { treatment field }\end{array}$ & -0.16 & -0.20 & -0.14 & 0.14 & 0.04 \\
\hline $\begin{array}{l}\text { Certified in an addiction } \\
\text { specialty }\end{array}$ & 0.09 & 0.20 & -0.04 & -0.11 & 0.07 \\
\hline $\begin{array}{l}\text { I would recommend this } \\
\text { treatment center to } \\
\text { colleagues. }\end{array}$ & $0.53^{* * *}$ & $0.59^{* * *}$ & $0.47^{* *}$ & $-0.43^{* *}$ & $-0.34^{*}$ \\
\hline $\begin{array}{l}\text { I have a good } \\
\text { relationship with the } \\
\text { clinical staff of this } \\
\text { organization. }\end{array}$ & $0.62^{* * *}$ & $0.63^{* * *}$ & $0.41^{*}$ & $-0.45^{* *}$ & -0.28 \\
\hline $\begin{array}{l}\text { I feel overburdened with } \\
\text { my current clinical } \\
\text { responsibilities at this } \\
\text { organization. }\end{array}$ & -0.24 & -0.25 & $-0.38^{*}$ & 0.31 & 0.27 \\
\hline $\begin{array}{l}\text { I feel valued and } \\
\text { respected by this } \\
\text { organization. }\end{array}$ & $0.36^{*}$ & 0.27 & 0.13 & $-0.35^{*}$ & -0.23 \\
\hline
\end{tabular}

${ }^{*} p<.05,{ }^{* *} p<.01,{ }^{* * *} p<.001$ (two-tailed tests) 
organizational environment were re-examined. Of the eight variables, four were significantly associated with overall buprenorphine work satisfaction. Prescribers reporting a greater number of patients $(r=.46, p=$ .006), those who would recommend the center to others $(r=.56, p<.001)$, and those reporting positive relationships with staff $(r=.56, \mathrm{p}<.001)$ reported significantly greater overall buprenorphine work satisfaction. Prescribers who more strongly endorsed feeling overburdened reported lower overall buprenorphine satisfaction $(r=-.37, p=.02)$.

Table 3 presents bivariate correlations between the satisfaction items for XR-naltrexone work, patient volume, prescriber characteristics, and perceptions of the organizational environment. Overall, there were few significant correlations. The current number of XRnaltrexone patients was only correlated with the negative valence items, such that prescribers with more patients reported significantly lower levels of frustration and unmet expectations. Tenure in the addiction field was negatively associated with being pleased with and being satisfied with XR-naltrexone work, such that prescribers with greater tenure reported lower satisfaction. None of the organizational environment items were correlated with the items for XR-naltrexone work satisfaction. When the five items were combined into an overall scale of XR-naltrexone work satisfaction, none of the variables were significantly associated with this scale.

\section{Discussion}

This study examined prescribers' satisfaction with providing two types of MOUD, buprenorphine and XRnaltrexone. We found that prescribers are generally satisfied with providing these two MOUDs, which is important since prescriber dissatisfaction can lead to turnover and impact intentions to leave the profession $[34,35]$. Ratings for the two MOUDs were remarkably similar, which was somewhat surprising given the induction challenges [26] and shorter average retention that occurs with extended-release naltrexone [15, 27]. However, the correlates of satisfaction differed between the two medications, in that patient volume and some of the organizational context measures were associated with buprenorphine work satisfaction, but none of the variables were associated with XR-naltrexone work satisfaction.

One notable finding was that the number of patients was positively correlated with prescriber satisfaction with delivering buprenorphine treatment. This magnitude of the association between census and the scale of buprenorphine satisfaction represented an effect size in the range of medium to large [36]. Questions remain about the direction of causality. It may be that treating more patients is an indicator of proficiency in or penchant for delivering this type of MOUD, which may positively impact satisfaction in treating this population. In other types of medical care, such as surgical procedures, a

Table 3 Pearson's product-moment correlations between patient volume, prescriber characteristics, organizational environment, and injectable naltrexone satisfaction

\begin{tabular}{|c|c|c|c|c|c|}
\hline & $\begin{array}{l}\text { I find my present } \\
\text { injectable naltrexone } \\
\text { clinical work } \\
\text { personally rewarding. }\end{array}$ & $\begin{array}{l}\text { Overall, I am } \\
\text { pleased with my } \\
\text { injectable } \\
\text { naltrexone work. }\end{array}$ & $\begin{array}{l}\text { Overall I am satisfied } \\
\text { with my current } \\
\text { injectable naltrexone } \\
\text { practice. }\end{array}$ & $\begin{array}{l}\text { My current injectable } \\
\text { naltrexone work is a } \\
\text { major source of } \\
\text { frustration. }\end{array}$ & $\begin{array}{l}\text { My work in this } \\
\text { injectable naltrexone } \\
\text { practice has not met } \\
\text { my expectations. }\end{array}$ \\
\hline $\begin{array}{l}\text { Current number of } \\
\text { injectable naltrexone } \\
\text { patients }\end{array}$ & 0.26 & 0.17 & 0.17 & $-0.40^{*}$ & $-0.37^{*}$ \\
\hline $\begin{array}{l}\text { Tenure in addiction } \\
\text { treatment field }\end{array}$ & -0.27 & $-0.45^{*}$ & $-0.38^{*}$ & -0.03 & 0.31 \\
\hline $\begin{array}{l}\text { Certified in an addiction } \\
\text { specialty }\end{array}$ & 0.21 & 0.19 & 0.01 & -0.29 & 0.05 \\
\hline $\begin{array}{l}\text { I would recommend } \\
\text { this treatment center to } \\
\text { colleagues. }\end{array}$ & 0.23 & 0.14 & -0.06 & -0.29 & -0.10 \\
\hline $\begin{array}{l}\text { I have a good } \\
\text { relationship with the } \\
\text { clinical staff of this } \\
\text { organization. }\end{array}$ & 0.24 & 0.15 & -0.09 & -0.32 & -0.13 \\
\hline $\begin{array}{l}\text { I feel overburdened } \\
\text { with my current clinical } \\
\text { responsibilities at this } \\
\text { organization. }\end{array}$ & 0.11 & 0.01 & 0.08 & 0.02 & -0.04 \\
\hline $\begin{array}{l}\text { I feel valued and } \\
\text { respected by this } \\
\text { organization. }\end{array}$ & -0.08 & -0.08 & -0.12 & -0.15 & -0.07 \\
\hline
\end{tabular}

${ }^{*} p<.05,{ }^{* *} p<.01,{ }^{* * *} p<.001$ (two-tailed tests) 
positive relationship between patient volume and patient outcomes is well-established [37, 38]. Treating more patients also provides more opportunities to observe patients entering remission and experiencing positive changes in their lives, which may also be a source of satisfaction. It may also be that prescribers who are more satisfied are more willing to take on additional patients. Future research should aim to replicate this finding in a larger sample with longitudinal data to help to better understand this association.

For buprenorphine work satisfaction, the associations regarding positive relationships with clinical staff and overly burdensome work demands align with prior research on physician satisfaction [29-31]. These correlations represented medium to large effect sizes [36].What is puzzling, however, was that these measures of the organizational context were not associated with XRnaltrexone work satisfaction. One potential explanation may be sample size, in that fewer prescribers offered XR-naltrexone than buprenorphine. However, it may also be that there are differences in how these two types of MOUD are implemented in clinical settings, which may then have implications for if or how the organizational context may matter in terms of prescriber satisfaction. Future research using qualitative semistructured in-depth interviews with prescribers may be an important method for better understanding how organizational context intersects with satisfaction in delivering different types of MOUD. In the current study, qualitative data collection focused on the issue of MOUD capacity-building from the perspective of organizational champions of MOUD who were most often in administrative positions [39]. The qualitative interviews revealed novel facilitators and barriers that differentiated organizations that made continuous improvements in MOUD capacity from those that did not. This ability to identify innovative factors inductively is a key strength of qualitative methodology and points to how in-depth interviews with prescribers could similarly lead to novel insights regarding satisfaction with delivering MOUD.

The study also provides insights into how the provision of MOUD might impact prescriber retention patterns. For prescribers, higher volumes of buprenorphine patients correlated with higher satisfaction levels. Simply encouraging prescribers to prescribe more buprenorphine will not likely lead to higher satisfaction by itself. Future research should seek to explore what leads to an individual physician wanting to prescribe buprenorphine to more patients and to create those working conditions that could lead to greater use of buprenorphine as well as greater satisfaction. For example, if a prescriber is expressing concerns regarding their confidence in using buprenorphine, there may be value in engaging them with an Extension for Community Healthcare Outcomes (ECHO) session or a peer mentor. The positive correlation between strong relationships with other clinical team members and buprenorphine satisfaction may indicate that a multi-discipline care model helps to make delivering buprenorphine treatment more meaningful and less burdensome. Our findings about $\mathrm{XR}$-naltrexone satisfaction and tenure in the field suggest that perhaps use of XR-naltrexone could be encouraged for prescribers who are earlier in their career. For those more advanced in their career, it may be that additional supports are needed to assist them with using this MOUD and appreciating its benefits, as those with greater tenure in the addiction field reported lower XR-naltrexone satisfaction.

Although this sample of prescribers is small, the findings aligned with a number of studies that have reported that most prescribers of buprenorphine are not fully utilizing their capacity based on their waiver type [14, 4044]. A recent analysis documented that a small subset of buprenorphine prescribers $(\sim 5 \%)$ were responsible for nearly half of US prescriptions over a two year period, and even these high volume prescribers averaged about 124 patients per month, which was well below the 275 patient cap [45]. In this sample, prescribers were on average using only about one-third of their capacity. Prescribers treated even fewer patients with injectable naltrexone, a finding that is consistent with other studies that have examined insurance claims data on utilization [15]. Unused MOUD treatment capacity is concerning given the scope of the ongoing opioid epidemic. Additional research is needed to further elucidate the factors associated with low utilization.

Several limitations to this study should be noted. First, the sample was quite small, and the prescribers were recruited from only three states. Our data collection strategy relied upon a key point of contact forwarding the survey invitations to prescribers, making it difficult to ascertain a response rate. A separate survey of organizational characteristics indicated a total of 65 waivered prescribers worked in these organizations, and we received responses from 38 waivered prescribers (58\%). It is unknown if dissatisfied prescribers were less likely to respond to the survey. Also, the findings are from a cross-sectional survey which cannot establish causality. In addition, this study was conducted in the context of an RCT focused on expanding MOUD, so the organizations recruited as sites may have cultures that are more supportive of MOUD which may have implications for physician satisfaction. Finally, only a limited number of constructs were included in the survey. Additional domains may be associated with prescriber satisfaction, such as challenges in obtaining reimbursement 
from insurers and stress experiences from concerning patient behaviors (e.g., misuse and diversion of buprenorphine, treatment dropout). Personality factors may also be correlated with prescriber satisfaction. Future research should also consider whether some of the factors that are associated with general job satisfaction are also correlated with prescribers' satisfaction in providing MOUD.

While future research should examine these issues in a larger, more generalizable sample, the study points to potentially important directions for future research. Continuing to examine additional factors associated with prescriber satisfaction with MOUD may identify directions for intervention development, including strategies to increase MOUD use and foster prescriber retention. Furthermore, qualitative data collection may help to elucidate why the factors associated with satisfaction seemed to differ between the two types of medication.

\section{Conclusions}

In the context of the opioid epidemic, there are ongoing concerns of shortages of prescribers of MOUD [46-48], so satisfaction among those providing these evidencebased practices is important. This sample of prescribers reported general satisfaction with delivering buprenorphine and XR-naltrexone, and their ratings did not differ between these two types of MOUD. There may be value in encouraging prescribers with small caseloads to consider expanding their MOUD practice, as our findings suggest there may be a benefit for prescriber satisfaction from treating more patients with MOUD, although more research is needed to confirm this finding. Managers of clinics should attend to supporting a positive organizational climate, as measures of organization context were associated with satisfaction in delivering buprenorphine. Future research is needed to examine multivariate models of satisfaction, to determine whether MOUD dissatisfaction is associated with prescribers' deimplementing this service, and to develop interventions that may reduce dissatisfaction.

\section{Abbreviations}

COVID-19: coronavirus disease; ECHO: Extension for Community Healthcare Outcomes; MOUD: medication for opioid use disorder; OUD: opioid use disorder; RCT: randomized clinical trial; XR: extended-release

\section{Acknowledgements}

The study team gratefully acknowledges the contributions of the prescribers who completed the survey.

\section{Authors' contributions}

HKK conceived the research question, conducted the statistical analyses, drafted the initial manuscript, and contributed to revisions. TM is the principal investigator of the study, who reviewed literature as well as contributed to drafting and revising the manuscript. JH recruited participants and contributed to drafting and revising the manuscript. RB, NJ, HK, JK, EC, $\mathrm{LM}$, and $\mathrm{EH}$ contributed to revisions of the manuscript. All authors read and approved the final manuscript.

\section{Funding}

This study was supported by a grant from the National Institute on Drug Abuse (NIDA Grant R01DA041415; PI: Molfenter). NIDA has played no role in the study design or preparation of this manuscript. The authors are solely responsible for the content of this manuscript, and this manuscript does not represent the official views of the National Institutes of Health or NIDA.

\section{Availability of data and materials}

Given the small sample size, the dataset for the current study is not publicly available in an effort to protect participant confidentiality. However, they are available from the corresponding author on reasonable request.

\section{Declarations}

Ethics approval and consent to participate

The study was approved by the Institutional Review Board of the University of Wisconsin-Madison (2016-0486).

\section{Consent to publication}

Not applicable.

Competing interests

The authors declare that they have no competing interests.

\section{Author details}

${ }^{1}$ Department of Behavioral Science and Center on Drug and Alcohol Research, University of Kentucky, 845 Angliana Ave., Room 204, KY 40508 Lexington, USA. ${ }^{2}$ Department of Family Medicine and Community Health, University of Wisconsin-Madison, 1100 Delaplaine Ct, WI 53715-1896 Madison, USA. ${ }^{3}$ Institute for Clinical and Translational Research, University of Wisconsin-Madison, 4116 Signe Skott Cooper Hall, 701 Highland Ave, WI 53705 Madison, USA. ${ }^{4}$ Department of Industrial and Systems Engineering, University of Wisconsin-Madison, 1513 University Ave, WI 53706 Madison, USA. ${ }^{5}$ Department of Educational Psychology, University of Wisconsin-Madison, 1025 West Johnson St, WI 53706-1706 Madison, USA ${ }^{6}$ Department of Internal Medicine, Yale University, APT Foundation, 1 Long Wharf Drive, Suite 321, CT 06511 New Haven, USA. ${ }^{7}$ Haram Consulting, 413 River Road, ME 04008 Bowdoinham, USA.

Accepted: 29 September 2021

Published online: 18 October 2021

\section{References}

1. Wilson N, Kariisa M, Seth P, Smith Ht, Davis NL. Drug and opioid-involved overdose deaths - United States, 2017-2018. MMWR Morb Mortal Wkly Rep. 2020;69:290-7.

2. Alexander GC, Stoller KB, Haffajee RL, Saloner B. An epidemic in the midst of a pandemic: Opioid use disorder and COVID-19. Ann Intern Med. 2020;173: 57-8.

3. Wainwright JJ, Mikre M, Whitley P, Dawson E, Huskey A, Lukowiak A, et al. Analysis of drug test results before and after the us declaration of a national emergency concerning the COVID-19 outbreak. JAMA. 2020.

4. Slavova S, Rock P, Bush HM, Quesinberry D, Walsh SL. Signal of increased opioid overdose during COVID-19 from emergency medical services data. Drug Alcohol Depend. 2020;214:108176.

5. American Society of Addiction Medicine. The ASAM National Practice Guideline for the Use of Medications in the Treatment of Addiction Involving Opioids Chevy Chase. MD: ASAM; 2015.

6. Substance Abuse and Mental Health Services Administration. Medications for opioid use disorder (Treatment Improvement Protocol (TIP) Series 63). Rockville: SAMHSA; 2018.

7. Volkow ND, Frieden TR, Hyde PS, Cha SS. Medication-assisted therapies-Tackling the opioid-overdose epidemic. N Engl J Med. 2014;370:2063-6.

8. Volkow ND, Collins FS. The role of science in addressing the opioid crisis. N Engl J Med. 2017:377:391-4.

9. Volkow ND. Medications for opioid use disorder: bridging the gap in care. Lancet. 2018:391:285-7.

10. Dick AW, Pacula RL, Gordon AJ, Sorbero M, Burns RM, Leslie D, et al. Growth in buprenorphine waivers for physicians increased potential access to opioid agonist treatment, 2002-11. Health Aff. 2015;34:1028-34. 
11. Andrilla CHA, Moore TE, Patterson DG, Larson EH. Geographic distribution of providers with a DEA waiver to prescribe buprenorphine for the treatment of opioid use disorder: A 5-year update. J Rural Health. 2019;35:108-12.

12. Knudsen HK, Lin LA, Lofwall MR. Adoption of the 275-patient buprenorphine treatment waiver for treating opioid use disorder: A statelevel longitudinal analysis. Subst Abus. 2020;41:259-68.

13. Auty SG, Stein MD, Walley AY, Drainoni ML. Buprenorphine waiver uptake among nurse practitioners and physician assistants: The role of existing waivered prescriber supply. J Subst Abuse Treat. 2020;115:108032.

14. Duncan A, Anderman J, Deseran T, Reynolds I, Stein BD. Monthly patient volumes of buprenorphine-waivered clinicians in the US. JAMA Netw Open. 2020;3:e2014045.

15. Morgan JR, Schackman BR, Leff JA, Linas BP, Walley AY. Injectable naltrexone, oral naltrexone, and buprenorphine utilization and discontinuation among individuals treated for opioid use disorder in a United States commercially insured population. J Subst Abuse Treat. 2018; 85:90-6.

16. Boudreau DM, Lapham G, Johnson EA, Bobb JF, Matthews AG, McCormack J, et al. Documented opioid use disorder and its treatment in primary care patients across six U.S. health systems. J Subst Abuse Treat. 2020;112S:41-8.

17. Becker WC, Fiellin DA. Provider satisfaction with office-based treatment of opioid dependence: a systematic review. Subst Abus. 2005;26:15-22.

18. Andrilla CHA, Moore TE, Patterson DG. Overcoming barriers to prescribing buprenorphine for the treatment of opioid use disorder: Recommendations from rural physicians. J Rural Health. 2019;35:113-21.

19. Knudsen HK, Brown R, Jacobson N, Horst J, Kim JS, Collier E, et al. Physicians' satisfaction with providing buprenorphine treatment. Addict Sci Clin Pract. 2019;14:34.

20. Williams ES, Skinner AC. Outcomes of physician job satisfaction: a narrative review, implications, and directions for future research. Health Care Manage Rev. 2003;28:119-39.

21. Sinsky CA, Dyrbye LN, West CP, Satele D, Tutty M, Shanafelt TD. Professional satisfaction and the career plans of US physicians. Mayo Clin Proc. 2017;92: 1625-35.

22. Williams ES, Konrad TR, Scheckler WE, Pathman DE, Linzer M, McMurray JE, et al. Understanding physicians' intentions to withdraw from practice: the role of job satisfaction, job stress, mental and physical health. Health Care Manage Rev. 2001;26:7-19.

23. Linn LS, Brook RH, Clark VA, Davies AR, Fink A, Kosecoff J. Physician and patient satisfaction as factors related to the organization of internal medicine group practices. Med Care. 1985;23:1171-8.

24. Haas JS, Cook EF, Puopolo AL, Burstin HR, Cleary PD, Brennan TA. Is the professional satisfaction of general internists associated with patient satisfaction? J Gen Intern Med. 2000;15:122-8.

25. DiMatteo MR, Sherbourne CD, Hays RD, Ordway L, Kravitz RL, McGlynn EA, et al. Physicians' characteristics influence patients' adherence to medical treatment: results from the Medical Outcomes Study. Health Psychol. 1993; 12:93-102.

26. Lee JD, Nunes EV Jr, Novo P, Bachrach K, Bailey GL, Bhatt S, et al. Comparative effectiveness of extended-release naltrexone versus buprenorphine-naloxone for opioid relapse prevention (X:BOT): a multicentre, open-label, randomised controlled trial. Lancet. 2018;391: 309-18.

27. Jarvis BP, Holtyn AF, Subramaniam S, Tompkins DA, Oga EA, Bigelow GE, et al. Extended-release injectable naltrexone for opioid use disorder: a systematic review. Addiction. 2018;113:1188-209.

28. Morgan JR, Schackman BR, Weinstein ZM, Walley AY, Linas BP. Overdose following initiation of naltrexone and buprenorphine medication treatment for opioid use disorder in a United States commercially insured cohort. Drug Alcohol Depend. 2019;200:34-9.

29. Hall CB, Brazil K, Wakefield D, Lerer T, Tennen H. Organizational culture, job satisfaction, and clinician turnover in primary care. J Prim Care Community Health. 2010;1:29-36.

30. McMurray JE, Williams E, Schwartz MD, Douglas J, Van Kirk J, Konrad TR, et al. Physician job satisfaction: developing a model using qualitative data. SGIM Career Satisfaction Study Group. J Gen Intern Med. 1997;12:711-4.

31. Linzer M, Sinsky CA, Poplau S, Brown R, Williams E. Healthy Work Place I. Joy in medical practice: Clinician satisfaction in the Healthy Work Place Trial. Health Aff (Millwood). 2017;36:1808-14.

32. Molfenter $\mathrm{T}$, Knudsen $\mathrm{H}$, Brown $\mathrm{R}$, Jacobsen $\mathrm{N}$, Horst J, Van Etten M, et al. Test of a workforce development intervention to expand opioid use disorder treatment pharmacotherapy prescribers: Protocol for a clusterrandomized trial. Implement Sci. 2017:12:135. https://implementa tionscience.biomedcentral.com/articles/https://doi.org/10.1186/s13012-0170665-X.

33. Williams ES, Konrad TR, Linzer M, McMurray J, Pathman DE, Gerrity M, et al. Refining the measurement of physician job satisfaction: results from the Physician Worklife Survey. SGIM Career Satisfaction Study Group. Society of General Internal Medicine. Med Care. 1999;37:1140-54.

34. Miller J. Workforce survey 2018. Behavioral Healthcare Executive 2018: Available from: https://www.behavioral.net/article/management/workforcesurvey-2018.

35. Shanafelt TD, Boone S, Tan L, Dyrbye LN, Sotile W, Satele D, et al. Burnout and satisfaction with work-life balance among US physicians relative to the general US population. Arch Intern Med. 2012;172:1377-85.

36. Cohen J. A power primer. Psychol Bull. 1992;112:155-9.

37. Chowdhury MM, Dagash H, Pierro A. A systematic review of the impact of volume of surgery and specialization on patient outcome. Br J Surg. 2007; 94:145-61.

38. Morche J, Mathes T, Pieper D. Relationship between surgeon volume and outcomes: a systematic review of systematic reviews. Syst Rev. 2016;5:204.

39. Jacobson N, Horst J, Wilcox-Warren L, Toy A, Knudsen HK, Brown R, et al. Organizational facilitators and barriers to medication for opioid use disorder capacity expansion and use. J Behav Health Serv Res. 2020;47:439-48.

40. Huhn AS, Dunn KE. Why aren't physicians prescribing more buprenorphine? J Subst Abuse Treat. 2017;78:1-7.

41. Kermack A, Flannery M, Tofighi B, McNeely J, Lee JD. Buprenorphine prescribing practice trends and attitudes among New York providers. J Subst Abuse Treat. 2017;74:1-6.

42. Thomas CP, Doyle E, Kreiner PW, Jones CM, Dubenitz J, Horan A, et al. Prescribing patterns of buprenorphine waivered physicians. Drug Alcohol Depend. 2017;181:213-8.

43. Stein BD, Sorbero M, Dick AW, Pacula RL, Burns RM, Gordon AJ. Physician capacity to treat opioid use disorder with buprenorphine-assisted treatment. JAMA. 2016;316:1211-2.

44. Andrilla CHA, Coulthard C, Patterson DG. Prescribing practices of rural physicians waivered to prescribe buprenorphine. Am J Prev Med. 2018;54: 208-S14.

45. Stein BD, Saloner B, Schuler MS, Gurvey J, Sorbero M, Gordon AJ. Concentration of Patient Care Among Buprenorphine-Prescribing Clinicians in the US. JAMA. 2021;325:2206-8.

46. Stein BD, Gordon AJ, Dick AW, Burns RM, Pacula RL, Farmer CM, et al. Supply of buprenorphine waivered physicians: The influence of state policies. J Subst Abuse Treat. 2015;48:104-11.

47. Knudsen HK, Havens JR, Lofwall MR, Studts JL, Walsh SL. Buprenorphine physician supply: Relationship with state-level prescription opioid mortality. Drug Alcohol Depend. 2017;173(Suppl 1):55-64.

48. Jones CM, Campopiano M, Baldwin G, McCance-Katz E. National and state treatment need and capacity for opioid agonist medication-assisted treatment. Am J Public Health. 2015;105:e55-63.

\section{Publisher's Note}

Springer Nature remains neutral with regard to jurisdictional claims in published maps and institutional affiliations.

Ready to submit your research? Choose BMC and benefit from:

- fast, convenient online submission

- thorough peer review by experienced researchers in your field

- rapid publication on acceptance

- support for research data, including large and complex data types

- gold Open Access which fosters wider collaboration and increased citations

- maximum visibility for your research: over $100 \mathrm{M}$ website views per year

At $\mathrm{BMC}$, research is always in progress.

Learn more biomedcentral.com/submissions 